\title{
Reduced-Order Modelling of Vortex-Induced Vibration of Catenary Riser
}

\author{
Narakorn Srinil $^{\mathrm{a}, \mathrm{b}}{ }^{*}$, Marian Wiercigroch $^{\mathrm{a}}$, Patrick O'Brien ${ }^{\mathrm{b}}$ \\ ${ }^{a}$ Centre for Applied Dynamics Research, School of Engineering, University of Aberdeen, \\ King's College, Scotland UK, ${ }^{b}$ MCS, Aberdeen, Scotland UK
}

\begin{abstract}
A new reduced-order model capable of analyzing the vortex-induced vibration of catenary riser in the ocean current has been developed. This semi analytical-numerical approach is versatile and allows for a significant reduction in computational effort for the analysis of fluid-riser interactions. The incoming current flow is assumed to be steady, uniform, unidirectional and perpendicular to the riser plane of initial equilibrium curvature. The equations of riser 3-D motion are based on a pinned-pinned, tensioned-beam or flexural cable, modelling which accounts for overall effects of riser bending, extensibility, sag, inclination and structural nonlinearities. The unsteady hydrodynamic forces associated with cross-flow and in-line vibrations are modelled as distributed van der Pol wake oscillators. This hydrodynamic model has been modified in order to capture the effect of varying initial curvature of the inclined flexible cylinder and to describe the space-time fluctuation of lift and drag forces. Depending on the vortex-excited in-plane/out-ofplane modes and system fluid-structure parameters, the parametric studies are carried out to determine the maximum response amplitudes of catenary risers, along with the occurrence of unimodal lock-in phenomenon. The obtained results highlight the effect of initial curvatures on the nonlinear dynamics of riser undergoing vortex-induced vibration.
\end{abstract}

Key Words: Reduced-Order Modelling, Vortex-Induced Vibration, Catenary Riser, Wake Oscillator, Fluid-Structure Interaction

* Corresponding Author: narakorn.srinil@abdn.ac.uk 


\section{INTRODUCTION}

Steel catenary risers (SCRs) are widely used in offshore installation, exploration and production. Because of their promising technological and commercial solutions, SCRs have become primary candidates for future ultra deepwater oil \& gas industry. One of the key issues in the analysis and design of SCRs in the ocean current is to estimate and control the fatigue damage due to vortexinduced vibration (VIV). Nevertheless, recent understanding of VIV is still based on empirical and/or simplified linearized models of straight top-tensioned risers (TTRs). Due to the impracticality of time-consuming 3-D flow visualizations by CFD (Computational Fluid Dynamics) for a long slender structure, the VIV fluid excitation and damping forces usually rely on the hydrodynamics data obtained from a laboratory testing of an elastically-supported rigid cylinder vibrating with 1 or 2 degree-of- freedom (DOF) in a uniform flow at low Reynolds (Re) numbers. Therefore, many uncertainties arise when designing the SCRs which are actually flexible inclined cylinders having initial sags and varying curvatures. As a matter of fact, SCRs are substantially different from TTRs, e.g., in view of the current flow direction relative to the pipe axis. The incident angle is arbitrary and different from $90^{\circ}$ when the flow aligns with the SCR plane of curvature. Moreover, a slender long sagged structure exhibits the cable-dominated behavior and has multiple natural frequencies which, in turn, potentially give rise to different inplane/out-of-plane modal interactions occurring in cross-flow/in-line VIV.

Nowadays, numerous frequency and time domain analysis tools for predicting nonlinear dynamic responses of risers undergoing VIV are available in industry. In spite of this, the stateof-the-art comparisons of VIV responses still exhibit significant discrepancies, even in the case of TTRs, between theoretical predictions and experimental measurements (Larsen and Halse 1997, Chaplin et al. 2005). More importantly, not much is actually known about VIV behaviour of SCRs. A prediction of fatigue damage of SCR has been shown by Vandiver and Gonzalez (1997) based on the combined use of mode superimposition and frequency domain approach. It has been realized that higher mode contributions are quite important to the damage rate. Due to the lack of empirical data on VIV of inclined and curved cylinders, the STRIDE joint industrial project has been initiated (Willis and Thethi 1999) to perform advanced testing on the VIV of towed curved pipes with/without strakes in water-tank and open-water environment. The corresponding experiment data have subsequently been considered by Moe et al. (2004) in order to validate the theoretical finite-element modelling. Because the experiment provides highly amplitudemodulated signals whereas the analysis tool provides steady-state responses, they have encountered the difficulty in making a direct comparison between analytical and experimental results even in the case of uniform flow. As a result, some discrepancies occurred and a number 
of experimental observations could not be theoretically explained. Lie et al. (2001) pointed out the significance of using the time domain approach with the inclusion of structural nonlinearities in the VIV analysis of SCRs.

Recently, an investigation into vortex shedding patterns and fundamental wake topology when the flow past a stationary curved circular cylinder has been carried out by Miliou et al. (2003, 2007). As a result of pipe curvatures, the computational simulations highlight different kinds of wake characteristics depending on the pipe (convex or concave) configuration and its orientation with respect to (aligned with or normal to) the incoming flow. When the flow is uniform and normal to the curvature plane, the cross-flow wake dynamics of curved pipes behave qualitatively similar to those of straight pipes. This is in contrast to the case of flow being aligned with the curvature plane where wake dynamics change dramatically. For this reason, in our initial study, the current flow approaching the SCR is assumed to be steady, unidirectional, uniform and perpendicular to the curvature plane of inclined cylinder (see Fig. 1). This avoids the multiplicity of vortex shedding frequencies (e.g., the case of flow aligned with the curvature plane) which would complicate the modelling and analysis.

Herein, the main emphasis is kept to evaluate the cross-flow and in-line VIV of SCRs due to fluctuating lift and drag forces, respectively. The paper is structured as follows. In Section 2, a general realistic fluid-structure interaction model for SCR (as well as TTR) with arbitrary sag and inclination is developed. The geometrically nonlinear equations of riser 3-D motion are derived accounting for both bending and tension rigidities. The catenary riser static configuration and corresponding modal shape functions are approximated by the hyperbolic and sine-series continuous functions, respectively. To model complex hydrodynamics where dynamic fluid forces are spatially distributed on the underwater cylindrical body, a phenomenological approach is used where spatially wake oscillators with relevant empirical coefficients are implemented. By coupling the wake oscillators to the riser equations governing in-plane and out-of-plane motions, a reduced-order model for the analysis of hydro/elastic-cylinder interaction due to uni-modal VIV is obtained in Section 3 and then systematically investigated in Section 4. The main objectives are (i) to determine the occurrence of lock-in phenomenon when varying the reduced flow velocity parameter and to predict, as well as validate, the associated maximum response amplitudes of SCRs, (ii) to investigate the effect of overall fluid-structure parameters and (iii) to highlight the significance of riser initial curvatures and riser geometric nonlinearities. 


\section{FLUID-RISER INTERACTION MODEL}

With reference to the global Cartesian coordinate system, Figure 1 depicts a general 3-D continuum model of SCR connected from a stationary floating structure to a seabed with simply pinned-pinned supports. A horizontal offset $X_{H}$ and water depth $Y_{H}$ define a chord inclination angle of riser (i.e., $\theta_{r}=\tan ^{-1} Y_{H} / X_{H}$ ). Riser properties are considered to be spatially uniform with mass/length $(m)$, viscous damping coefficient $(c)$, hydrodynamic diameter $(D)$, effective bending $(E I)$ and axial $\left(E A_{r}\right)$ stiffness. The steady incoming uniform flow, having density $(\rho)$ and normal velocity $(V)$, is in the $Z^{+}$-direction perpendicular to the SCR plane $(X Y)$ of initial equilibrium curvatures. Following the Strouhal number $(\mathrm{St})$ law of a stationary cylinder, the flow entails a single natural frequency $(\mathrm{rad} / \mathrm{s})$ of vortex shedding in the wake $\left(\omega_{s}\right)$, i.e., $\omega_{s}=2 \pi \mathrm{St} V / D$, where $\mathrm{St}$ $\approx 0.2$ being assumed for a sub-critical flow condition with $300<\operatorname{Re}<3 \times 10^{5}$ (e.g., Sumer and Fredsoe 1997).

\subsection{Geometrically Nonlinear Equations of Riser Motion}

In deepwater applications, the riser has a large aspect (length-to-diameter) ratio and is usually predominated by the tension behavior. Therefore, by considering the riser as a flexural sagged cable-like elastic structure satisfying the Euler-Bernoulli beam hypothesis, the nonlinear partialdifferential equations of riser motion about its planar $(X Y)$ static equilibrium configuration may be expressed in a general dimensional form as (Srinil et al. 2007; Ricciardi and Saitta 2008)

$$
\begin{aligned}
& \left(m+m_{a}\right) \frac{\partial^{2} u}{\partial t^{2}}+c \frac{\partial u}{\partial t}=\frac{\partial}{\partial s}\left\{T\left(\frac{\partial u}{\partial s}\right)+E A_{r}\left(\frac{\partial x}{\partial s} \frac{\partial u}{\partial s}+\frac{\partial y}{\partial s} \frac{\partial v}{\partial s}+\right.\right. \\
& \left.\left.\frac{1}{2}\left(\left(\frac{\partial u}{\partial s}\right)^{2}+\left(\frac{\partial v}{\partial s}\right)^{2}+\left(\frac{\partial w}{\partial s}\right)^{2}\right)\right)\left(\frac{\partial x}{\partial s}+\frac{\partial u}{\partial s}\right)-E I \frac{\partial}{\partial s}\left(\frac{\partial^{2} x}{\partial s^{2}}+\frac{\partial^{2} u}{\partial s^{2}}\right)\right\}+F_{1} \\
& \left(m+m_{a}\right) \frac{\partial^{2} v}{\partial t^{2}}+c \frac{\partial v}{\partial t}=\frac{\partial}{\partial s}\left\{T\left(\frac{\partial v}{\partial s}\right)+E A_{r}\left(\frac{\partial x}{\partial s} \frac{\partial u}{\partial s}+\frac{\partial y}{\partial s} \frac{\partial v}{\partial s}+\right.\right. \\
& \left.\left.\frac{1}{2}\left(\left(\frac{\partial u}{\partial s}\right)^{2}+\left(\frac{\partial v}{\partial s}\right)^{2}+\left(\frac{\partial w}{\partial s}\right)^{2}\right)\right)\left(\frac{\partial y}{\partial s}+\frac{\partial v}{\partial s}\right)-E I \frac{\partial}{\partial s}\left(\frac{\partial^{2} y}{\partial s^{2}}+\frac{\partial^{2} v}{\partial s^{2}}\right)\right\}+F_{2} \\
& \left(m+m_{a}\right)^{2} \frac{\partial^{2} w}{\partial t^{2}}+c \frac{\partial w}{\partial t}=\frac{\partial}{\partial s}\left\{T\left(\frac{\partial w}{\partial s}\right)+E A_{r}\left(\frac{\partial x}{\partial s} \frac{\partial u}{\partial s}+\frac{\partial y}{\partial s} \frac{\partial v}{\partial s}+\right.\right. \\
& \left.\left.\frac{1}{2}\left(\left(\frac{\partial u}{\partial s}\right)^{2}+\left(\frac{\partial v}{\partial s}\right)^{2}+\left(\frac{\partial w}{\partial s}\right)^{2}\right)\right)\left(\frac{\partial w}{\partial s}\right)-E I \frac{\partial}{\partial s}\left(\frac{\partial^{2} w}{\partial s^{2}}\right)\right\}+F_{3}
\end{aligned}
$$

in which $s$ denotes Lagrangian or arc-length coordinate and $t$ denotes time. $u(x) v(y)$ and $w$ 
represent global dynamic (static) displacement in the horizontal $(X)$, vertical $(Y)$ and transversal or out-of-plane $(Z)$ direction, respectively. $T$ denotes axial static tension of riser due to effective weight, $m_{a}$ denotes potential added mass $\left(C_{A} \rho A_{r}\right.$, where $A_{r}$ is circular cross-sectional area, $\left.C_{A}=1\right)$, and $F_{i}$ denotes external hydrodynamic (lift/drag) forces leading to VIV. By accounting for both bending (Ricciardi and Saitta, 2008) and axial (e.g., Srinil et al. 2007) rigidities, Equations (1)-(3) are also valid for a top-tensioned riser (TTR), mooring line or horizontal pipeline with zero sag. Overall inertia effects and structural nonlinearities (Srinil and Rega, 2007b), which are meaningful in the case of large dynamic displacement or deformation, are fully accounted for. It is worth mentioning that the effects of shear, torsion, seabed interaction and internal-flowinduced friction forces, which are quite important for SCRs, are not herein considered. In the following, all the space-related variables and associated equations are non-dimensionalized with respect to $D$.

For convenience in our analytical modelling, a planar submerged static configuration of SCR solely due to effective self weight is assumed, whereas the bending restraint and the uniform current flow play a role after the performance of static equilibrium. The neglected static bending is plausible because the end boundaries are pinned-pinned and the SCR curvatures are relatively small. Accordingly, the higher-order spatial derivatives of $x$ and $y$ in Eqs. (1) and (2) are disregarded, and the static profile of SCR is simply governed by

$$
\frac{T_{H}}{D} y^{\prime \prime}=-W_{E} \sqrt{1+y^{\prime 2}}
$$

in which a dash denotes differentiation with respect to the new space variable $x, W_{E}$ is the computed effective weight accounting for buoyancy effect, and $T_{H}$ is a horizontal component of riser tension which is spatially constant. By directly integrating Eq. (4) twice, the exact hyperbolic function-based formula describing the catenary configuration reads

$$
y(x)=\frac{-T_{H}}{W_{E} D} \cosh \left(\frac{-W_{E} D}{T_{H}} x+C_{1}\right)+C_{2},
$$

where $C_{1}$ are $C_{2}$ can be determined based on boundary conditions. Thus, for a given $D, m, \theta_{r}, X_{H}$ and $T_{H}$, the SCR equilibrium can be explicitly determined and then substituted into Eqs. (1)-(3) as an embedded continuous function.

As the current flow is normal to the SCR plane, the cross-flow (in-line) VIV due to lift $F_{L}$ (drag $F_{D}$ ) force corresponds to in-plane (out-of-plane) motion of SCR. By neglecting the tangential hydrodynamics, the excitation forces per unit length in Eqs. (1)-(3) read 


$$
\begin{aligned}
& F_{1}=-F_{L} \sin \theta=-\frac{1}{2} \rho C_{L} D V^{2} \sin \theta, \\
& F_{2}=F_{L} \cos \theta=\frac{1}{2} \rho C_{L} D V^{2} \cos \theta, \\
& F_{3}=F_{D}=\frac{1}{2} \rho C_{D} D V^{2},
\end{aligned}
$$

where $\theta$ is a local angle of inclination (measured clockwise from the $X$-axis in Fig. 1) based on Eq. (5), in which $\theta \approx \tan ^{-1}\left(y^{\prime}\right) . C_{L}(s, t)$ and $C_{D}(s, t)$ are unsteady lift and drag coefficients per unit length, respectively. It is worth noting that the mean drag, and possibly also the mean lift (Miliou et al. 2003, 2007), component, which potentially gives rise to a new SCR equilibrium, is here omitted as we focus on the fluctuating component.

\subsection{Nonlinear Wake Oscillators}

To model the unsteady hydrodynamic forces as a result of vortex shedding in the cylinder wake, a nonlinear wake oscillator proves to be quite simple and the most computationally-effective means (Gabbai and Benaroya 2005). This wake oscillator is scrutinized as simplified, phenomenological and semi-empirical model which provides the analyst an understanding of some fundamental nonlinear phenomena of VIV, in spite of having no involvement in the flow physical characterization. Some ad hoc assumptions are common in analytical models. These comprise, e.g., the consideration of nominal two-dimensional flow at all times, the full correlation length of vortex shedding along the cylinder length (i.e., under lock-in condition) and the neglected effects of end boundaries and stream-wise cylinder movement. To produce a benchmarking series of empirical wake coefficients vs. physical parameters, rigid and elastically-mounted cylinders in a uniform flow have been mostly considered in experimental campaigns. A wake oscillator is typically based on the nonlinear van der Pol equation having a term (terms) coupled with a structural equation of motion such as the linear spring-mass-damping oscillator. Almost all of the models reported in literature to date are restricted to lift forces governing cross-flow VIV, with the measurement set-up being usually rearranged such that the drag force governing in-line VIV is negligible or uncoupled with cross-flow VIV. Nevertheless, the wake oscillator proves to be quite useful for describing a self-limiting nature of VIV responses observed by many experiments and flow visualizations. In addition, the lock-in or synchronization regime can be analyzed along with the prediction of corresponding response amplitudes. Recently, the van der Pol oscillator for VIV has been revised by Skop and Balasubramanian SB (1997), Facchinetti, de Langre and 
Biolley FLB (2004) in order to overcome some limitations of previous wake oscillators. Both models capture the self-limiting amplitude responses at zero structural damping and reproduce some qualitative, as well as quantitative, aspects of VIV when compared with experiment results. Regarding the application to flexible cylinders, the SB model has been used in the analysis of single-mode cross-flow/in-line VIV of horizontally suspended cables by Kim and Perkins (2002). The FLB model has been considered by Violette et al. (2007) for the cross-flow VIV of long straight tensioned-beam and cable based on a linear structural model. They showed a good comparison with direct numerical simulations and experiments.

In this study, the SB model is considered. The distributed lift coefficient $C_{L}(s, t)$ and associated wake oscillator $Q(s, t)$ are originally expressed as

$$
\begin{aligned}
& C_{L}(s, t)=Q(s, t)-\frac{2 \gamma}{\omega_{s}} \dot{Y}_{N}(s, t), \\
& \ddot{Q}(s, t)-\omega_{s} \bar{G}\left(C_{L 0}^{2}-4 Q^{2}(s, t)\right) \dot{Q}(s, t)+\omega_{s}^{2} Q(s, t)=\omega_{s} \bar{F} \dot{Y}_{N}(s, t),
\end{aligned}
$$

where $\gamma$ is a so-called stall parameter (Triantafyllou et al. 1994), $Y_{N}$ is defined as a local riser displacement normal to its tangential axis, $C_{L 0}$ is a given lift coefficient of a stationary cylinder, and a dot denotes differentiation with respect to time. Wake coefficients are $\bar{F}$ and $\bar{G}$, with the overbar denoting "empirical" quantity. To account for the effect of SCR initial curvature and also describe the concurrent horizontal/vertical displacement components of SCR in-plane motion, we project $Q_{X}=-Q \sin \theta$ and $Q_{Y}=Q \cos \theta$, by using the cosine law. This entails two new wake oscillators

$$
\begin{gathered}
\frac{\ddot{Q}_{X}}{\sin \theta}-\frac{\omega_{s} \bar{G} C_{L 0}^{2} \dot{Q}_{X}}{\sin \theta}+\frac{4 \omega_{s} \bar{G} Q_{X}^{2} \dot{Q}_{X}}{\sin ^{3} \theta}+\frac{\omega_{s}^{2} Q_{X}}{\sin \theta}=\frac{\omega_{s} \bar{F} \dot{u}}{\sin \theta} \\
\frac{\ddot{Q}_{Y}}{\cos \theta}-\frac{\omega_{s} \bar{G} C_{L 0}^{2} \dot{Q}_{Y}}{\cos \theta}+\frac{4 \omega_{s} \bar{G} Q_{Y}^{2} \dot{Q}_{Y}}{\cos ^{3} \theta}+\frac{\omega_{s}^{2} Q_{Y}}{\cos \theta}=\frac{\omega_{s} \bar{F} \dot{v}}{\cos \theta} .
\end{gathered}
$$

In contrast to Eq. (10), Eqs. (11) and (12) are now dependent on both time and space variables. $Q_{X}$ and $Q_{Y}$ are to be determined together with $u$ and $v$. The wake coefficients $(\bar{F}, \bar{G})$ are obtained by matching a series of experimental data which generally include the measurement of maximum response amplitude of cylinder $A / D$ and vortex-to-structural frequency ratio during VIV (Skop and Balasubramanian 1997). As exemplified in Fig. 2, $\bar{F}$ and $\bar{G}$ are dependent on the system mass-damping (the so-called Skop-Griffin) parameter $\mathrm{S}_{\mathrm{G}}=\xi / \mu$, in which $\xi$ is the riser modal damping and $\mu$ is the mass ratio given by 


$$
\mu=\frac{\rho D^{2}}{8 \pi^{2} \mathrm{St}^{2}\left(m+m_{a}\right)} .
$$

As for in-line VIV, very few theoretical studies have proposed a wake oscillator governing the drag coefficient, and a practical tool for predicting the in-line VIV is still unavailable in industry. However, it is well known from many experiments (e.g., Okajima et al. 2004) that in-line VIV may take place in a reduced velocity range lower than that of cross-flow VIV with symmetric/alternate vortices. In addition, it may take place in the same reduced velocity range as cross-flow VIV with alternate vortices. Typically, the in-line VIV has a frequency twice that of cross-flow VIV during a complete 2-D lock-in. This entails that both out-of-plane and in-plane modes, whose natural frequencies are in nearly-tuned 2:1 ratio, are simultaneously excited. Based on this evidence and also considering the practical case of alternate vortices, we assume, by following Currie and Turnbull (1987), Kim and Perkins (2002),

$$
\begin{aligned}
& C_{D}(s, t)=P(s, t)+\frac{1}{2 \omega_{s}} \dot{P}(s, t)-\frac{2 \gamma}{\omega_{s}} \dot{w}(s, t), \\
& \ddot{P}(s, t)-2 \omega_{s} \bar{H}\left(C_{D 0}^{2}-4 P^{2}(s, t)\right) \dot{P}(s, t)+4 \omega_{s}^{2} P(s, t)=2 \omega_{s} \bar{J} \dot{w}(s, t),
\end{aligned}
$$

where $P$ is the in-line wake component, $\bar{H}$ and $\bar{J}$ are empirical coefficients, and $C_{D 0}$ is the drag coefficient of the stationary cylinder.

It is worth emphasizing that Eqs.(6) and (11) (7 and 12) are dependent on both $Q_{X}$ and $u\left(Q_{Y}\right.$ and $v$ ), exhibiting the two-way feedback coupled system of the wake-riser interactions. This holds also for Eqs.(8) and (15) involving $P$ and $w$. In place of Eqs. (11) and (12), Eq. (10) is considered for cross-flow VIV of a straight vertical riser or horizontal pipe.

\subsection{Riser Natural Frequencies and Modes with Bending-Tension Effect}

In the framework of analytical modelling, the natural frequencies and mode shapes of pinnedpinned SCRs (as well as TTRs) have to be determined in terms of continuous functions. In so doing, the linear equations of undamped free in-plane $(u, v)$ and out-of-plane $(w)$ motion, corresponding to Eqs.(1)-(3), are considered and given in a dimensionless form by

$$
\kappa \ddot{u}+b^{2}\left(\frac{1}{\kappa^{3}} u^{\prime \prime \prime}\right)^{\prime}-c^{2}\left\{\left(1+\frac{\alpha}{\kappa^{3}}\right) u^{\prime}+\left(\frac{\alpha y^{\prime}}{\kappa^{3}}\right) v^{\prime}\right\}^{\prime}=0,
$$




$$
\begin{aligned}
& \kappa \ddot{v}+b^{2}\left(\frac{1}{\kappa^{3}} v^{\prime \prime \prime}\right)^{\prime}-c^{2}\left\{\left(\frac{\alpha y^{\prime}}{\kappa^{3}}\right) u^{\prime}+\left(1+\frac{\alpha y^{\prime 2}}{\kappa^{3}}\right) v^{\prime}\right\}^{\prime}=0, \\
& \kappa \ddot{w}+b^{2}\left(\frac{1}{\kappa^{3}} w^{\prime \prime \prime}\right)^{\prime}-c^{2} w^{\prime \prime}=0,
\end{aligned}
$$

where $\kappa=\left(1+y^{\prime 2}\right)^{1 / 2}, \alpha=E A_{r} / T_{H}, b^{2}=E I /\left(m+m_{a}\right) D^{4}, c^{2}=T_{H} /\left(m+m_{a}\right) D^{2}$. With zero displacements and bending moments at the end boundaries, the in-plane and out-of-plane modes are postulated in terms of a Fourier sine-based series as

$$
U^{J}(x, t)=\sum_{n=1}^{N} \Upsilon_{n}^{J}(t) \sin \left(\frac{n \pi x D}{X_{H}}\right)
$$

where, for $J=1-3, U^{1}=u, U^{2}=v, U^{3}=w, \Upsilon_{n}^{J}$ are generalized time coordinates, $N$ is the number of retained sine functions. By substituting Eq. (19) into Eqs. (16)-(18) and applying the Galerkin method, the eigenvalue problem is solved via a hybrid analytical-numerical solution proposed by Srinil et al. (2007). Note also that, for a pinned-pinned TTR with uniform tension and bending, both frequencies and modal shapes can be alternatively obtained via closed-form formulae, with both $k^{\text {th }}$ in-plane/out-of-plane modes being similar to taut-string modes. Nevertheless, this is not the case for SCRs, whose in-plane modes are significantly dependent on initial sagged and curved configurations. These modes are indeed neither purely symmetric nor anti-symmetric due to the effect of geometric asymmetry.

To understand the global in-plane/out-of-plane frequency relationships when varying some key parameters of the riser, it is worth constructing a spectrum of natural frequencies in still water. This is useful in view of detecting the potential cross-flow/in-line VIV modes. Due to the combined effect of riser bending, extensibility (axial tension) and geometry (sag/inclination), a single dimensionless tensioned-beam parameter is introduced, namely

$$
\Delta=L \sqrt{T_{a} / E I}
$$

where $L$ is the riser equilibrium length and $T_{a}$ is the tension at maximum sag of SCR (or the averaged tension in the case of TTR). This parameter describes how the flexural ( $\operatorname{small} \Delta$ ) or axial (large $\Delta$ ) rigidity plays a predominant role. By normalizing the obtained SCR frequencies $(\omega)$ by the lowest frequency of the corresponding TTR $\left(\omega_{T}\right)$, the natural frequency spectrum of tensiondominated risers is illustrated in Fig. 3 with $\omega / \omega_{T}$ vs. $\Delta$ and solid (dotted) lines denoting in-plane (out-of-plane) modes. 
In Fig. 3, $T_{a}$ (also $L$ ) is varied while keeping $E I$ fixed. $\Delta>560$ represents the case of TTRs (as sag $\rightarrow 0$, giving rise to the negligible $W_{E}$ effect with respect to $T_{a}$ ). The $k$ in-plane/out-of-plane frequency ratio is apparently equal to 1 (like taut-string frequencies). However, when decreasing $T_{a}$ (increasing sag), the lower $\Delta(\Delta<560)$ reflects the case of SCRs whose $k$ in-plane/out-of-plane frequency ratio is different from 1 . In some circumstances, the vortex frequency $\omega_{s}$ (for a given $V$ ) may simultaneously excite the in-line/cross-flow VIV modes having a 2:1 frequency ratio, as exemplified by the circles corresponding to the $6^{\text {th }}$ out-of-plane $/ 2^{\text {nd }}$ in-plane modes for $\Delta \approx 272$ (vertical dashed line). With a further increase of $V$ (thus $\omega_{s}$ ) for such $\Delta$, it is also possible that two higher in-plane $\left(5^{\text {th }}\right.$ and $\left.6^{\text {th }}\right)$ modes - whose frequencies are nearly equal at a so-called avoidance region (Srinil and Rega, 2007a) - can be both excited. This may result in a multi-mode lock-in of cross-flow VIV (Hover et al. 1997). Yet, our attention is focused on the uni-modal lock-in behavior which will be analyzed through a reduced-order model derived in the following section.

\section{REDUCED-ORDER MODEL FOR CROSS-FLOW AND IN-LINE VIV}

With the aim of minimizing the computational effort, a reduced-order model describing the hydrodynamics-elastic cylinder interaction is now developed. The first-order (i.e. state-space) differential forms of Eqs. (1)-(3), (11), (12) and (15) are considered, and the expansion of displacement and velocity (defined by $A_{i}, B_{i}$ ) variables is postulated by the following forms,

$$
\begin{aligned}
& \dot{u}=A_{1} \rightarrow \quad u=\sum_{m=1}^{\infty} f_{m} \phi_{m}, \quad A_{1}=\sum_{m=1}^{\infty} p_{m} \phi_{m}, \\
& \text { For riser dynamics: } \quad \dot{v}=A_{2} \rightarrow \quad v=\sum_{m=1}^{\infty} f_{m} \varphi_{m}, \quad A_{2}=\sum_{m=1}^{\infty} p_{m} \varphi_{m} \text {, } \\
& \dot{w}=A_{3} \rightarrow \quad w=\sum_{n=1}^{\infty} h_{n} \zeta_{n}, \quad A_{3}=\sum_{n=1}^{\infty} q_{n} \zeta_{n} . \\
& \dot{Q}_{x}=B_{1} \rightarrow \quad Q_{x}=\sum_{m=1}^{\infty} d_{m} \phi_{m}, \quad B_{1}=\sum_{m=1}^{\infty} e_{m} \phi_{m},
\end{aligned}
$$

For wake dynamics: $\quad \dot{Q}_{y}=B_{2} \rightarrow \quad Q_{y}=\sum_{m=1}^{\infty} d_{m} \varphi_{m}, \quad B_{2}=\sum_{m=1}^{\infty} e_{m} \varphi_{m}$,

$$
\dot{P}=B_{3} \rightarrow \quad P=\sum_{n=1}^{\infty} z_{n} \zeta_{n}, \quad B_{3}=\sum_{n=1}^{\infty} o_{n} \zeta_{n} .
$$

where $\phi_{m}$ and $\varphi_{m}$ represent the horizontal and vertical component of $m^{\text {th }}$ in-plane (cross-flow VIV) modal shape function, $\zeta_{n}$ represents the $n^{\text {th }}$ out-of-plane (in-line VIV) modal shape function, $f_{m}\left(d_{m}\right), p_{m}\left(e_{m}\right), h_{n}\left(z_{n}\right)$, and $q_{n}\left(o_{n}\right)$ are the corresponding generalized coordinates of riser (wake) to be determined. By further assuming that both the fluid in the form of a distributed wake 
oscillator and the riser dynamically respond in a similar fashion having a spatial shape profile corresponding to a potentially vortex-induced mode, the number of considered in-plane (out-ofplane) modes is equal to 1. This assumption is plausible (see, e.g., Skop and Griffin, 1975; Kim and Perkins, 2002) as the flow is uniform and its direction is perpendicular to the SCR in-plane curvature, giving rise to a single vortex shedding frequency. Moreover, because the VIV amplitude is relatively small with respect to $D$ (Sarpkaya 2004), contributions from higher-order modes through structural nonlinearities may be negligible (Srinil and Rega, 2007b). By substituting Eqs. (21) and (22) into Eqs. (1)-(3), (11), (12) and (15), applying the Galerkin method with relevant boundary conditions and orthonormalization of modal shapes, a set of nonlinearly coupled equations, governing a single in-plane/out-of-plane mode VIV response and fulfilling the 2-D lock-in (i.e. $\omega_{n} \approx 2 \omega_{m} \approx 2 \omega_{s}$ ) condition, is obtained as

$$
\begin{aligned}
& \dot{f}_{m}=p_{m}, \\
& \dot{p}_{m}=-2 \mu \omega_{m}\left(\mathrm{~S}_{\mathrm{G} m}+\gamma\right) p_{m}-\omega_{m}^{2} f_{m}+\Lambda_{m} f_{m}^{2}+\vartheta_{m n} h_{n}^{2}+\Pi_{m} f_{m}^{3}+\Upsilon_{m n} f_{m} h_{n}^{2}+\mu \omega_{m}^{2} d_{m}, \\
& \dot{h}_{n}=q_{n}, \\
& \dot{q}_{n}=-2 \mu \omega_{n}\left(\mathrm{~S}_{\mathrm{G} n}+\gamma / 2\right) q_{n}-\omega_{n}^{2} h_{n}+\Psi_{n m} h_{n} f_{m}+\Delta_{n m} h_{n} f_{m}^{2}+\Theta_{n} h_{n}^{3}+\mu \omega_{n}^{2} z_{n} / 4+\mu \omega_{n} o_{n} / 4, \\
& \dot{d}_{m}=e_{m}, \\
& \dot{e}_{m}=\omega_{m} \bar{G} C_{L 0}^{2} e_{m}-\frac{4 \omega_{m} \bar{G}}{\Gamma_{m}} e_{m} d_{m}^{2}-\omega_{s}^{2} d_{m}+\omega_{m} \bar{F} p_{m}, \\
& \dot{z}_{n}=o_{n}, \\
& \dot{o}_{n}=\omega_{n} \bar{H} C_{D 0}^{2} o_{n}-\frac{4 \omega_{n} \bar{H}}{\Gamma_{n}} o_{n} z_{n}^{2}-4 \omega_{s}^{2} z_{n}+\omega_{n} \bar{J} q_{n},
\end{aligned}
$$

where the modal shape-based quadratic and cubic nonlinear coefficients are given by

$$
\begin{aligned}
& \Lambda_{m}=\frac{-3 c^{2} \alpha}{2} \int_{0}^{X_{H} / D} \frac{1}{\kappa^{3}}\left(\phi_{m}^{\prime 3}+y^{\prime} \phi_{m}^{\prime 2} \varphi_{m}^{\prime}+\phi_{m}^{\prime} \varphi_{m}^{\prime 2}+y^{\prime} \varphi_{m}^{\prime 3}\right) d x, \\
& \vartheta_{m n}=\frac{-c^{2} \alpha}{2} \int_{0}^{X_{H} / D} \frac{1}{\kappa^{3}}\left(\phi_{m}^{\prime} \zeta_{n}^{\prime 2}+y^{\prime} \varphi_{m}^{\prime} \zeta_{n}^{\prime 2}\right) d x, \\
& \Pi_{m}=\frac{-c^{2} \alpha}{2} \int_{0}^{X_{H} / D} \frac{1}{\kappa^{3}}\left(\phi_{m}^{\prime 4}+2 \phi_{m}^{\prime 2} \varphi_{m}^{\prime 2}+\varphi_{m}^{\prime 4}\right) d x, \\
& \Upsilon_{m n}=\frac{-c^{2} \alpha}{2} \int_{0}^{X_{H} / D} \frac{1}{\kappa^{3}}\left(\phi_{m}^{\prime 2} \zeta_{n}^{\prime 2}+\varphi_{m}^{\prime 2} \zeta_{n}^{\prime 2}\right) d x,
\end{aligned}
$$




$$
\begin{aligned}
& \Theta_{n}=\frac{-c^{2} \alpha}{2} \int_{0}^{X_{H} / D} \frac{1}{\kappa^{3}}\left(\zeta_{n}^{\prime 4}\right) d x, \\
& \Gamma_{m}=\int_{0}^{X_{H} / D} \kappa\left(\frac{\phi_{m}^{2}}{y^{\prime}}+\varphi_{m}^{2}\right) d x / \int_{0}^{X_{H} / D} \kappa^{3}\left(\frac{\phi_{m}^{4}}{y^{\prime 3}}+\varphi_{m}^{4}\right) d x, \\
& \Gamma_{n}=\int_{0}^{X_{H} / D} \zeta_{n}^{2} d x / \int_{0}^{X_{H} / D} \zeta_{n}^{4} d x .
\end{aligned}
$$

It can be analytically proved that $\Psi_{n m}=2 \vartheta_{m n}$ and $\Delta_{n m}=\Upsilon_{m n}$. Eqs.(31)-(37) are numerically integrated based on a 64-point Gaussian Quadrature. When considering a straight riser (or horizontal cable) involving an anti-symmetric mode in the VIV, some coefficients are trivial due to the nonlinear orthogonality of modal shape functions (Srinil and Rega 2007a). Depending on assigned initial conditions, empirical coefficients and fluid-structure parameters, Eqs.(23)-(30) are simultaneously solved by numerical integrations with a proper time stepping method. To perform parametric studies, it is worth making a reference to a reduced flow velocity parameter,

$$
U_{r}=\frac{2 \pi V}{\omega_{m} D}=\frac{1}{\mathrm{St} \delta}
$$

where $\delta=\omega_{m} / \omega_{s}$ being a reduced angular frequency of the riser. Here, $D$ is fixed, whereas $U_{r}$ is varied through the first or second relationship in Eq. (38). In the first relation, the flow velocity $V$ is varied whereas, in the second relation, the vortex frequency $\omega_{s}$ or the riser in-plane frequency $\omega_{m}$ is varied through $\delta$ while keeping $V(\mathrm{Re})$ fixed. Typically, for convenience in the experiments, $V$ is increased or decreased while keeping other properties of the tested cylinder fixed. Yet, for long flexible cylinders such as marine risers, the system frequencies $\left(\omega_{m}, \omega_{n}\right)$ may be closely spaced (e.g., Fig. 3) and, when varying such $V$, different potential modes may be excited according to the resultant shedding frequency $\omega_{s}$. Moreover, due to the associated variation of Re, the assumption of sub-critical flow in making use of the wake oscillator might not be valid when further increasing $V$. To circumvent this, the $V(\mathrm{Re})$ may be fixed by parametrically varying $\omega_{m}$ or $\omega_{s}$. If $\omega_{m}$ is varied, the so-called true, in-situ or oscillation frequency is realized as $\omega_{m} \pm \sigma$, where $\sigma$ is a cross-flow frequency detuning parameter. This variation is practically reasonable since the structural natural frequency during VIV is indeed modulated due to the varying added mass coefficient (Blevin 1990, Vandiver 1993). Alternatively, by keeping $\omega_{m}$ fixed, $\omega_{s}$ may be varied through $\omega_{s} \pm \varepsilon$, where $\varepsilon$ is a vortex frequency detuning parameter, since the vortex frequency of 
oscillating cylinder may be different from that of stationary cylinder given by Strouhal law. The variation of out-of-plane (or in-line VIV) frequency $\omega_{n}$ can be made in the same manner. When obtaining the steady-state solution of Eqs. (23)-(30), the temporally $\left(f_{m}\right.$ or $\left.h_{n}\right)$ and spatially ( $\square_{m}$, $\varphi_{m}$ or $\left.\zeta_{n}\right)$ maximum amplitudes due to cross-flow $\left(A_{m} / D\right)$ and in-line $\left(A_{n} / D\right)$ VIV can be deduced from the time histories in conjunction with Eq. (21). Namely,

$$
\begin{aligned}
& A_{m} / D=\operatorname{Max} \sqrt{\left(f_{m} \phi_{m}\right)^{2}+\left(f_{m} \varphi_{m}\right)^{2}}, \\
& A_{n} / D=\operatorname{Max}\left|h_{n} \zeta_{n}\right| .
\end{aligned}
$$

Because of solving for the temporal generalized coordinate $\left(f_{m}\right.$ or $\left.h_{n}\right)$ based on a single-mode VIV analysis, the spatial location of maximum VIV amplitude is the same as the anti-node position of the corresponding linear mode shape function $\left(\square_{m}, \varphi_{m}\right.$ or $\left.\zeta_{n}\right)$.

\section{PARAMETRIC INVESTIGATIONS}

As an example, we first consider a tension-dominated SCR with high aspect ratio $(L / D) \approx 2581$, $\theta_{r}=30^{\circ}, \alpha \approx 1669, b^{2} \approx 8.1 \times 10^{6}, c^{2} \approx 7.8 \times 10^{4}, \Delta \approx 272$ (corresponding to Fig. 3 ). The fluidstructure parameters are $\mu \approx 0.044, \mathrm{~S}_{\mathrm{G} m}=\mathrm{S}_{\mathrm{G} n} \approx 0.068$ (i.e. both in-plane/out-of-plane modal damping values are assumed to be equal, with $\xi=0.003), C_{L 0}=0.28, C_{D 0}=0.20, \gamma \approx 0.183, \bar{F} \approx$ 0.398 and $\bar{G} \approx 1.061$ (see Fig. 2). As analytical formulae for estimating the empirical wake coefficients of in-line VIV are unavailable in literature, we shall assume $\bar{J} \approx \bar{F}$ and $\bar{H} \approx \bar{G}$. For the given averaged $V=0.34 \mathrm{~m} / \mathrm{s}$, the computed $\omega_{s} \approx 1.112 \mathrm{rad} / \mathrm{s}$ and the potential cross-flow (inline) VIV mode corresponds to the $2^{\text {nd }}$ in-plane ( $6^{\text {th }}$ out-of-plane) mode (Fig. 3) with $\omega_{m} \approx 1.033$ $\left(\omega_{n} \approx 2.207\right) \mathrm{rad} / \mathrm{s}$. The associated normalized in-plane $(\phi, \varphi)$ and out-of-plane $(\zeta)$ modal shape functions projected onto the $X$-axis are displayed in Fig. 4 with convergent 40 sine functions (Srinil et al. 2007). These nearly-symmetric (4a) and anti-symmetric (4b) modes, together with above-mentioned parameters, are considered, unless stated otherwise, in the following parametric studies of uni-modal cross-flow/in-line VIV.

\subsection{Nonlinear Dynamic Interactions and Uni-Modal Lock-In Phenomenon}

Depending on assigned initial displacement/velocity conditions, the time histories associated with cross-flow $\left(f_{m}\right)$ and in-line $\left(h_{n}\right)$ displacement amplitudes are comparatively displayed in Fig. 5 with $U_{r} \approx 6$. It can be seen that, after some transient dynamics, the cross-flow VIV response (red line) reaches the steady state or "limit cycle" prior to the in-line VIV response (dashed blue line), with a slight phase difference between their time series. Depending on the mass and damping 
ratios, the phase angle between cross-flow and in-line VIV has recently been evidenced by an experiment study of elastically-mounted rigid cylinder vibrating with two degrees of freedom (Jauvtis and Williamson 2004). Overall dynamic responses in Fig.5 are perfectly periodic, with cross-flow displacement amplitudes being greater than in-line amplitudes (Sarpkaya 2004). In turn, the corresponding oscillating (in-line:cross-flow) frequencies are nearly tuned in a 2:1 ratio. A comparison of displacement responses governing the riser and the fluid wake force is displayed in Fig. 6a for cross-flow VIV $\left(f_{m}\right.$ vs. $\left.d_{m}\right)$ and in Fig. 6c for in-line VIV $\left(h_{n}\right.$ vs. $\left.z_{n}\right)$, along with the associated phase portraits $\left(f_{m}, p_{m}\right)$ and $\left(h_{n}, q_{n}\right)$ in Figs. $6 \mathrm{~b}$ and $6 \mathrm{~d}$, respectively. It is shown that a slight phase difference between wake and riser steady-state responses occurs more apparently in cross-flow VIV (Fig. 6a). The closed-orbit phase plots (6b and 6d) show the passage from transient to steady-state motion as well as the periodicity of limit cycle, with the cross-flow velocity (displacement) parameter $p_{m}\left(f_{m}\right)$ having smaller (larger) maximum amplitudes than the in-line velocity (displacement) parameter $q_{n}\left(h_{n}\right)$. Overall, Figs. 6 and 7 show the fundamental characteristics of uni-modal wake-riser interaction which involves a single-frequency, selflimiting, and steady-state response.

The typical lock-in or synchronization in which cross-flow and in-line VIV occur over a wide range of the reduced flow velocity $U_{r}$ is now highlighted, along with the predicted maximum response amplitudes $A / D$. As aforesaid through Eq. (38), $U_{r}$ can be altered by either varying $V, \omega_{s}$ or $\omega_{m}\left(\omega_{n}\right)$, which, in turn, parametrically affects Eqs. (23)-(30). For the sake of comparison, the results with varying $V$ and system frequencies are presented in Figs. 7 and 8, respectively. In Fig. 7 , the flow velocity is either increased or decreased in the range $0.1<V<0.7 \mathrm{~m} / \mathrm{s}\left(\approx 3.2 \times 10^{4}<\operatorname{Re}\right.$ $<2 \times 10^{5}$ ) with a small increment of $0.01 \mathrm{~m} / \mathrm{s}$. Both cross-flow and in-line VIV amplitudes are comparatively plotted versus $U_{r}$. It can be seen that a large $A / D$ variation due to cross-flow (inline) VIV occurs in the range $4<U_{r}<7\left(5<U_{r}<6\right)$, with the discontinuity of two response branches owing to a jump phenomenon or hysteresis effect. This highlights the lock-in phenomenon whereby the riser and the fluid are in the internally-resonant condition, with the vortex shedding frequency locking into the riser oscillation frequency (Sumer and Fredsoe 1999). When increasing or decreasing $V$, overall riser responses coincide: for the sudden jump-down and jump-up, critical $U_{r}$ values are nearly the same. The bent-to-right exhibits a hardening behavior likely due to the predominant cubic nonlinearities associated with wake oscillators. The greater response amplitudes - as well as the broader regime of lock-in - correspond to the cross-flow VIV giving rise to the maximum $A / D \approx 1.426$, in comparison with maximum $A / D \approx 0.779$ due to in-line VIV. These occur albeit assuming similar properties $(\bar{J} \approx \bar{F}, \bar{H} \approx \bar{G})$ for cross-flow and 
in-line VIV. Overall, the presented theoretical wake-riser interaction model provides good qualitative agreement with theoretical and experimental literature for cross-flow/in-line VIV, in view of maximum attainable amplitudes (up to $A / D \approx 2$ for flexible cylinders) and the uni-modal lock-in prediction (Blevin 1990, Sarpkaya 2004).

Considering now the fixed flow velocity $v=0.35 \mathrm{~m} / \mathrm{s}$ and with this assumption the vortexexcited modes are the same as in Fig. 4. By varying $\omega_{s}$ or $\omega_{m}\left(\omega_{n}\right)$ through the corresponding frequency detuning parameter $\varepsilon$ or $\sigma$ within the same range of $[-0.6,1.7]$, similar response diagrams exhibiting the lock-in phenomenon are obtained in Figs. 8a (cross-flow VIV) and 8b (in-line VIV). The in-line vibration response and the associated lock-in bandwidth (Fig. 8b) seem to be more sensitive to the frequency variation. Yet, overall achievable amplitudes when varying $\omega_{s}$ or $\omega_{m}\left(\omega_{n}\right)$ are comparable, being approximately equal to those predicted in Fig. 7 with varying $V$. This similarity of Figs. 7 and 8 may be attributed to that the lock-in occurs in the range about $4<U_{r}<7\left(5<U_{r}<6\right.$ or 8) with $1.3>\omega_{m} / \omega_{s}>0.7\left(2.2>\omega_{n} / \omega_{s}>1.7\right)$ for cross-flow (in-line) VIV, rather than being at $\omega_{m} / \omega_{s}=1\left(\omega_{n} / \omega_{s}=2\right)$ or $U_{r}=5$ for the stationary riser.

A comparison of VIV responses between SCR and TTR having the same flexural tensionedbeam parameter $\Delta \approx 272$ is now shown in Fig. 9 with the case of increasing $V$. From Fig. 3 , the potentially excited modes for TTR correspond to the $3^{\text {rd }}$ cross-flow $\left(\omega_{m} \approx 1.095 \mathrm{rad} / \mathrm{s}\right)$ and sixth in-line $\left(\omega_{n} \approx 2.194 \mathrm{rad} / \mathrm{s}\right)$ modes, whose shapes are perfectly symmetric and anti-symmetric with respect to middle span with three and six half-sine waves, respectively. Based on the same given fluid-structure parameters, empirical coefficients and initial conditions as before, the response comparison in Fig. 9a highlights that the cross-flow VIV of TTR entails smaller $A / D(\approx 1.262)$ with respect to the cross-flow VIV of SCR $(\approx 1.425)$. This occurs although the lock-in ranges and corresponding response jumps appear similar for both risers. Such predicted amplitude difference is attributed to the effect of riser geometry, namely the riser initial curvatures, because TTR (SCR) has zero (non-zero) sag and has one (two) displacement component in the cross-flow VIV. The cross-flow amplitudes of TTR tend to be comparable to those of straight spring-mounted cylinders reported in the literature. On the contrary, the in-line VIV amplitudes for both TTR and SCR in Fig. $9 \mathrm{~b}$ are nearly comparable $(A / D \approx 0.76)$. This is physically reasonable because the inline modes of SCR and TTR are the same $6^{\text {th }}$ mode (Fig. 4b) and the in-line VIV of SCR subject to flow normal to the curvature plane is not significantly affected by riser initial curvatures.

\subsection{Influence of System Parameters}

First, the effect of SCR geometrical nonlinearities on the prediction of cross-flow and in-line VIV 
responses is highlighted in Fig. 10. A beam-dominant SCR of Moe et al. (2004) is now considered, whose parameters are $L / D \approx 835, \theta_{r}=37.62^{\circ}, \alpha \approx 16832, b^{2} \approx 2.35 \times 10^{9}, c^{2} \approx 1.2 \times 10^{6}$, $\Delta \approx 21, \mu \approx 0.121, \mathrm{~S}_{\mathrm{G} m}=\mathrm{S}_{\mathrm{G} n} \approx 0.082, \bar{F} \approx 0.432$ and $\bar{G} \approx 0.982$. With the averaged $V=1 \mathrm{~m} / \mathrm{s}\left(\omega_{s}\right.$ $\approx 89.76 \mathrm{rad} / \mathrm{s})$, the potentially excited cross-flow and in-line modes are the $10^{\text {th }}$ in-plane $\left(\omega_{m} \approx\right.$ $82.78 \mathrm{rad} / \mathrm{s})$ and $15^{\text {th }}$ out-of-plane $\left(\omega_{n} \approx 169.35 \mathrm{rad} / \mathrm{s}\right)$ modes, respectively. This highlights the VIV at high-mode numbers. By considering either linear (omitted $f_{m}^{2}, h_{n}^{2}, f_{m}^{3}, h_{n}^{2}, h_{n} f_{m}, h_{n} f_{m}^{2}, h_{n}^{3}$ ) or nonlinear equations of riser motion (Eqs. 23-26), the associated maximum $A / D$ responses are comparatively plotted versus $U_{r}$ in Fig. 10 in the case of increasing $V$. Overall, the jump phenomena are observed by both linear/nonlinear models. However, the comparison reveals noticeable amplitude differences in both cross-flow (Fig. 10a) and in-line (Fig. 10b) VIV, though based on the same assigned initial conditions. The linear model substantially overestimates the $A / D$ during the lock-in. The predicted maximum cross-flow (in-line) $A / D$ amplitudes are about $1.13(0.52)$ and $0.69(0.42)$ by linear and nonlinear models, respectively. Moreover, the linear model neglects the hardening (bent-to-right) nonlinear effect. This highlights that the geometrical nonlinearities - which indeed play a crucial role in establishing a new riser equilibrium caused by mean drag - should be accounted for, at least, for the sake of quantitative correctness.

Next, it is interesting to understand how the vortex-excited modes having different spatial shapes affect the VIV responses. For the fixed $\Delta \approx 272$ (the SCR in Fig. 3), the cross-flow and inline VIV modes whose frequency values are in $1: 2$ ratio are the $1^{\text {st }}\left(2^{\text {nd }}, 3^{\text {rd }}, 4^{\text {th }}, \ldots\right)$ in-plane and $4^{\text {th }}\left(6^{\text {th }}, 8^{\text {th }}, 10^{\text {th }}, \ldots\right)$ out-of-plane modes, respectively. Note that, due to the SCR in-plane configuration, the spatial shape profiles of odd $\left(1^{\text {st }}, 3^{\text {rd }}\right)$ or even $\left(2^{\text {nd }}, 4^{\text {th }}\right)$ in-plane modes are not perfectly anti-symmetric or symmetric (e.g., see Fig. 4a), whereas the spatial shape profiles of even out-of-plane modes are perfectly anti-symmetric (e.g., see Fig. 4b). With the same given parameters and empirical coefficients, the analysis of lock-in regime is performed in the case of increasing $V$, and the maximum $A / D$ results are compared in Table 1 for different potentiallyexcited modes. It can be seen that the in-line $A / D$ amplitude tends to slightly change with the corresponding mode order. This is in contrast to the case of cross-flow VIV, where different excited in-plane modes entail different maximum $A / D$, depending on the horizontal/vertical shape functions affecting overall coefficients in Eqs (31)-(37). Again, such difference between crossflow/in-line VIV is due to the influence of initial sag or curvatures of SCR on the in-plane vibration. The even $\left(2^{\text {nd }}, 4^{\text {th }}\right)$ modes seem to be the most dangerous case for this SCR example.

In practical design, the inclinations and sags of SCRs are variable, depending on the geometry (e.g., water depth, horizontal offset, seabed-free length) and the stiffness (e.g., bending and axial 
rigidity). This, in turn, influences the beam-cable behaviour of risers. Herein, to appreciate the solely effect of riser sag on VIV, we now consider three SCRs having different tensioned-beam parameters $\Delta$ (Fig. 3). For the sake of comparison, the bending stiffness $E I$, the $2^{\text {nd }}$ in-plane (cross-flow) and $6^{\text {th }}$ out-of-plane (in-line) modes are fixed in the VIV analysis of each SCR. With increasing $V$, the predicted maximum $A / D$ during cross-flow and in-line lock-in are comparatively reported in Table 2. It can be seen that both maximum cross-flow and in-line amplitudes slightly increase with decreasing $\Delta$ or increasing sag-to-span ratio. This is physically reasonable because the larger sagged SCR is more slender and flexible, potentially leading to larger vortex-induced displacements.

The mass-damping parameter (e.g., $\mathrm{S}_{\mathrm{G}}$ ) also plays a significant role in the VIV analysis and prediction (Sarpkaya 2004) because it affects empirical coefficients, vortex-shedding modes (Williamson and Roshko, 1988) and overall VIV response behaviors. As a matter of fact, many experimental VIV studies of elastically-mounted rigid or long flexible cylinders subject to normal flow depend on the measured mass and damping values. Therefore, it is worth making a comparison of analysis results with a series of experimental data. In Fig. 11, we compare the predicted maximum amplitudes during lock-in with those gathered by Skop and Balasubramanian (1997), by reporting $2 A / D$ in the so-called Griffin plot (Williamson and Govardhan, 2004). Three $\mathrm{S}_{\mathrm{G}}$ values $(0.068,0.227,1.133)$ are considered for both SCRs $\left(\theta_{r}=30^{\circ}\right)$ and corresponding TTRs, and results of the $2^{\text {nd }}$ (SCRs) and $4^{\text {th }}$ (TTRs) cross-flow VIV modes are displayed. In addition, the predicted numerical results by a frequency-domain approach Shear7 (see, e.g., Yang et al. 2008) are also given, based on the program recommended parameters and with two types of lift coefficient data (named as $C_{L}$ type 1 and 2). It is noted that Shear7 does not consider in-line VIV and, in general, the Shear7 results with $C_{L^{-}}$-Type 1 provide a more conservative $A / D$ prediction than those with $C_{L}$-Type 2.

Overall, the maximum cross-flow amplitudes of both SCRs and TTRs decrease with increasing $\mathrm{S}_{\mathrm{G}}$. This is true if the structural damping or the structural mass increases (see, e.g., Khalak and Williamson, 1999). It is also worth mentioning that the corresponding in-line VIV response (not shown herein) significantly decreases as $\mathrm{S}_{\mathrm{G}}$ increases and it possibly disappears when further increasing $\mathrm{S}_{\mathrm{G}}$. For TTRs, the predicted cross-flow $2 A / D$ amplitudes provide good qualitative, and possibly quantitative, agreement with Shear7 as well as experimental $2 A / D$ amplitudes. With respect to literature, for instance, Moe and Overvik (1982) considered a riser based on an elastically-mounted rigid cylinder model and reported that, for $\mathrm{S}_{\mathrm{G}}=0.23,2 A / D \approx 2.18$, whereas our study predicts that, for $\mathrm{S}_{\mathrm{G}}=0.227,2 A / D \approx 2.17$. For SCRs, the inclined flexible cylinders 
with sags tend to have greater $2 A / D$ than straight cylinders such as TTRs, pivoted tubes, cantilevers or taut cables. The time-domain analysis with nonlinear wake oscillator seems to provide more conservative results for low $\mathrm{S}_{\mathrm{G}}$ parameters. This prediction needs further experimental confirmation based on real SCR vs. TTR measurement data, with the same controlled $\mathrm{S}_{\mathrm{G}}$ parameters and environmental flow conditions.

\section{CONCLUSIONS}

A computationally-robust reduced-order model capable of describing the fluid-catenary riser interaction due to VIV has been developed. The riser model is based on the geometrically nonlinear equations of 3-D motion of a pinned-pinned tensioned-beam or flexural cable subject to a steady uniform current flow whose direction is perpendicular to the riser plane of initial equilibrium curvatures. The hydrodynamic model is based on the distributed nonlinear wake oscillators describing the fluctuating lift and drag forces corresponding to cross-flow and in-line VIV, respectively. Overall effects of riser bending, extensibility, sag, inclination and in-plane/outof-plane modal coupling through structural nonlinearities are fully taken into account.

Parametric studies have been carried out by numerical integrations to evaluate the maximum response amplitudes due to cross-flow and in-line VIV. The wake-riser nonlinear dynamic interactions depend on the modal shape functions of vortex-excited in-plane/out-of-plane modes, the tensioned-beam (sag, inclination, bending or extensibility) properties, the fluid-structure (e.g., mass-damping, vortex/structural frequencies) parameters, the empirical wake coefficients and the assigned initial conditions. The obtained results highlight the occurrence of uni-modal lock-in when varying the reduced flow velocity parameter, along with some fundamental features of VIV.

The comparative analysis of catenary and straight top-tensioned risers has also been performed. The predicted maximum amplitudes due to cross-flow (in-line) VIV of catenary riser are greater than (nearly comparable to) those of straight riser. This may be attributed to the influence of initial curvatures of catenary riser. With respect to the cross-flow VIV, the amplitude results provide good qualitative, as well as quantitative, agreement with experimental data of rigid/flexible cylinders in literature and with results by a frequency-domain approach. In some cases, the effect of riser geometrical nonlinearities is pronounced.

Because of the capability of predicting the uni-modal lock-in regime and associated maximum amplitudes due to cross-flow and in-line VIV, the presented reduced-order hydrodynamics-riser interaction model and analysis may be extended to account for the case of multimode VIV. These are theoretically and practically meaningful in actual applications where the flow is not 
perpendicular to the catenary riser plane of curvature and/or the flow is spatially sheared. Moreover, the associated development of finite element-based modeling, in conjunction with the improvement of nonlinear wake oscillators based on a new series of experimental measurements or CFD analyses of curved-pipe VIV, looks very promising.

ACKNOWLEDGEMENT The authors gratefully acknowledge the financial support by the Knowledge Transfer Partnerships (KTP).

\section{REFERENCES}

Blevins, RD (1990). Flow-Induced Vibrations, Van Nostrand Reinhold, New York, USA.

Chaplin, JR, Bearman, PW, Cheng, Y, Fontaine, E, Graham, JMR, Herfjord, K, Huera Huarte, FJ, Isherwood, M, Lambrakos, K, Larsen, CM, Meneghini, JR, Moe, G, Pattenden RJ, Triantafyllou, MS, Willden, RHJ (2005). "Blind predictions of laboratory measurements of vortex-induced vibrations of a tension riser," Journal of Fluids and Structures 21, 25-40.

Currie, IG, Turnbull, DH (1987). "Streamwise oscillations of cylinders near the critical Reynolds number," Journal of Fluids and Structures 1, 185-196.

Facchinetti, M, de Langre, E, Biolley, F, (2004), "Coupling of structure and wake oscillators in vortexinduced vibrations," Journal of Fluids and Structures 19, 123-140.

Gabbai, RD, Benaroya, H, (2005). "An overview of modeling and experiments of vortex-induced vibration of circular cylinders," Journal of Sound and Vibration 282, 575-616.

Hatton, S, Willis, N, (1998), "Steel catenary risers for deepwater environments," in Proceedings of the OTC, Paper No.8607.

Hover, F, Davis, JT, Triantafyllou, MS (1997). "Vottex-induced vibration of marine cables: experiments using force feedback," Journal of Fluids and Structures 11, 307-326.

Jauvtis, N, Williamson, CHK (2004), "The effect of two degrees of freedom on vortex-induced vibration at low mass damping," Journal of Fluids Mechanics 509, 23-62.

Khalak, A, Williamson, CHK (1999). "Motions, forces and mode transitions in vortex-induced vibrations at low mass-damping," Journal of Fluids and Structures 13, 813-851.

Kim, WJ, Perkins, NC (2002). “Two-dimensional vortex-induced vibration of cable suspensions," Journal of Fluids and Structures 16, 229-245.

Larsen, CM, Halse, KH (1997). "Comparison of models for vortex-induced vibrations of slender marine structures," Marine Structures 10, 413-441.

Lie, H, Larsen, CM, Tveit, O, (2001), "Vortex induced vibration analysis of catenary riser," in Proceedings of the OTC, Paper No.13115.

Miliou, A, Sherwin, SJ, Graham, JM, (2003), "Fluid dynamic loading on curved riser pipes," Journal of 
Offshore Mechanics and Arctic Engineering 125, 176-182.

Miliou, A, De Vecchi, A, Sherwin, SJ, Graham, JMR (2007). "Wake dynamics of external flow past a curved circular cylinder with the free stream aligned with the plane of curvature," Journal of Fluids Mechanics 592, 89-115.

Moe, G, Overvik, T (1982). "Current-induced motions of multiple risers," Proceedings of BOSS-82, Behaviour Offshore Structures, ed. Chryyostomides, C, Conner, JJ, 618-639.

Moe, G, Teigen, T, Simantiras, P, Willis, N, Lie, H, (2004), "Predictions and model tests of a SCR undergoing VIV in flow at oblique angles," in Proceedings of the $23^{\text {rd }}$ OMAE, Vancouver, Paper No.51563.

Okajima, A, Nakamura, A, Kosugi, T, Uchida, H, Tamaki, R (2004). "Flow-induced in-line oscillation of a circular cylinder," European Journal of Mechanics B/Fluids 23, 115-125.

Ricciardi, G, Saitta, F (2008). "A continuous vibration analysis model for cables with sag and bending stiffness," Engineering Structures 30, 1459-1472.

Sarpkaya, T (2004). "A critical review of the intrinsic nature of vortex-induced vibrations," Journal of Fluids and Structures 19, 389-447.

Skop, RA, Balasubramanian, S (1997). "A new twist on an old model for vortex-excited vibrations," Journal of Fluids and Structures 11,395-412.

Skop, RA, Griffin, OM (1975). "On a theory for the vortex-excited oscillations of flexible cylindrical structures," Journal of Sound and Vibration 41, 263-274.

Srinil, N, Rega, G, Chucheepsakul, S (2007). "Two-to-one resonant multi-modal dynamics of horizontal/inclined cables. Part I: Theoretical formulation and model validation," Nonlinear Dynamics 48, 231-252.

Srinil, N, Rega, G (2007a). "Two-to-one resonant multi-modal dynamics of horizontal/inclined cables. Part II: Internal resonance activation, reduced-order models and nonlinear normal modes," Nonlinear Dynamics 48, 253-274.

Srinil, N, Rega, G (2007b). "The effects of kinematic condensation on internally resonant forced vibrations of shallow horizontal cables," International Journal of Nonlinear Mechanics 42, 180195.

Sumer, BM, Fredsoe, J (1997). Hydrodynamics around Cylindrical Structures, World Scientific, Singapore.

Triantafyllou, MS, Gopalkrishnan, R, Grosenbaugh, MA (1994). "Vortex-induced vibrations in a sheared flow: a new predictive method," In Hydroelasticity in Marine Technology, Rotterdam, 31-37.

Vandiver, JK (1993). "Dimensionless parameters important to the prediction of vortex-induced vibration of long, flexible cylinders in ocean currents," Journal of Fluids and Structures 7, 423-455.

Vandiver, JK, Gonzales, EC, (1997), "Fatigue life of catenary risers excited by vortex shedding," in 
Proceedings of the $8^{\text {th }}$ BOSS, Delft.

Violette, R, de Langre, E, Szydlowski, J, (2007). "Computation of vortex-induced vibration of long structures using a wake oscillator model: comparison with DNS and experiments," Computers and Structures 85, 1134-1141.

Williamson, CHK, Govardhan, R (2004). "Vortex-induced vibrations," Annual Review of Fluid Mechanics 36, 413-455.

Williamson, CHK, Roshko, A (1988). "Vortex formation in the wake of an oscillating cylinder," Journal of Fluids and Structures 2, 355-381.

Yang, G, Frank, WR, Campbell, RB, Slocum, ST (2008), "VIV model test data comparison with Shear7 V4.5," in Proceedings of the $27^{\text {th }}$ OMAE, Estoril, Paper No.57108. 


\section{$\underline{\text { List of Figures }}$}

Figure 1: A model of catenary riser subject to uniform current flow

Figure 2: $\quad$ Variation of empirical wake coefficients with mass-damping parameter $\mathrm{S}_{\mathrm{G}}$

Figure 3: $\quad$ Variation of riser natural frequencies with tensioned-beam parameter $\Delta$

Figure 4: $\quad$ Considered (a) $2^{\text {nd }}$ in-plane and (b) $6^{\text {th }}$ out-of-plane modes for cross-flow and in-line VIV, respectively

Figure 5: Time histories of riser displacement coordinates and associated with crossflow $\left(f_{m}\right.$, red solid line) and in-line $\left(h_{n}\right.$, blue dotted line) VIV

Figure 6: (a) Cross-flow VIV (solid line) and lift force (dotted line) responses, (b) Inline VIV (dashed line) and drag force (dotted line) responses, with corresponding phase portraits of riser motion $\left(f_{m}-p_{m}\right.$ and $\left.h_{n}-q_{n}\right)$ in (c) and (d)

Figure 7: Maximum amplitude responses due to cross-flow (circles, squares) and in-line (stars, triangles) VIV of SCR, with increasing $V$ (circles, stars) or decreasing $V$ (squares, triangles)

Figure 8: $\quad$ Maximum amplitude responses due to (a) cross-flow and (b) in-line VIV of SCR, with varying $\omega_{s}$ (filled symbols) or varying $\omega_{m}$ and $\omega_{n}$ (open symbols)

Figure 9: Maximum amplitude responses due to (a) cross-flow and (b) in-line VIV: SCR (filled symbols), TTR (open symbols)

Figure 10: Maximum amplitude responses due to (a) cross-flow and (b) in-line VIV of SCR, with geometrically nonlinear (filled symbols) and linear (open symbols) modelling

Figure 11: A comparison of maximum cross-flow VIV amplitudes during lock-in of TTR and SCR with some experimental data and Shear7 results

\section{List of Tables}

Table 1: A comparison of maximum $A / D$ due to cross-flow and in-line VIV for SCR with $\Delta=272$ involving different excited modes

Table 2: $\quad$ A comparison of maximum $A / D$ due to cross-flow and in-line VIV for SCRs having different tensioned-beam parameters $\Delta$ and sag-to-span ratios 
Fig.1

Click here to download Figure: Fig. 1.pdf

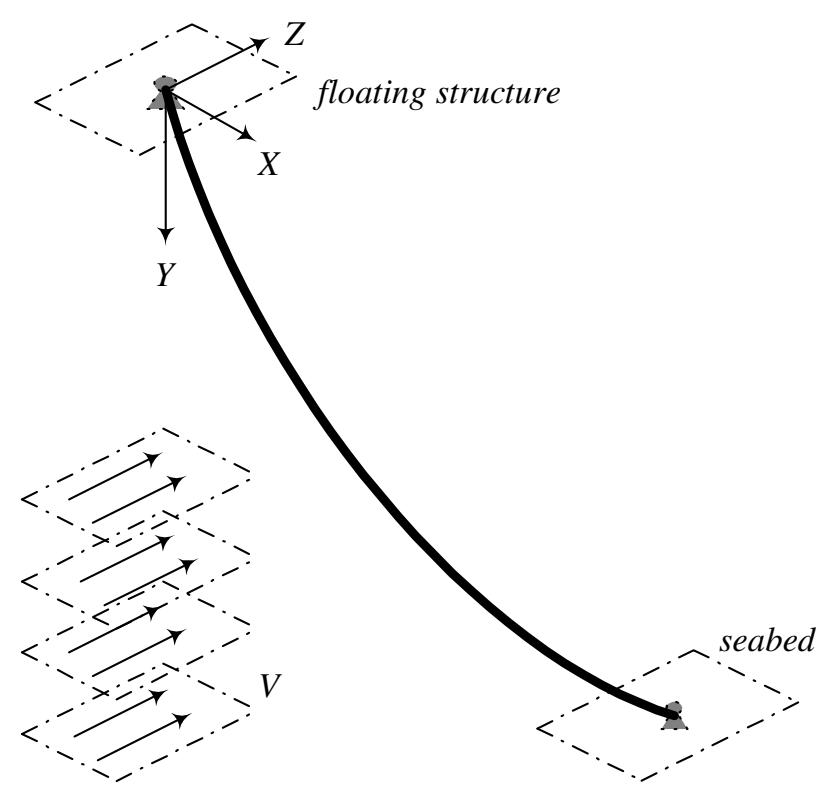

Figure 1 A model of catenary riser subject to uniform current flow 
Fig.2

Click here to download Figure: Fig. 2.pdf

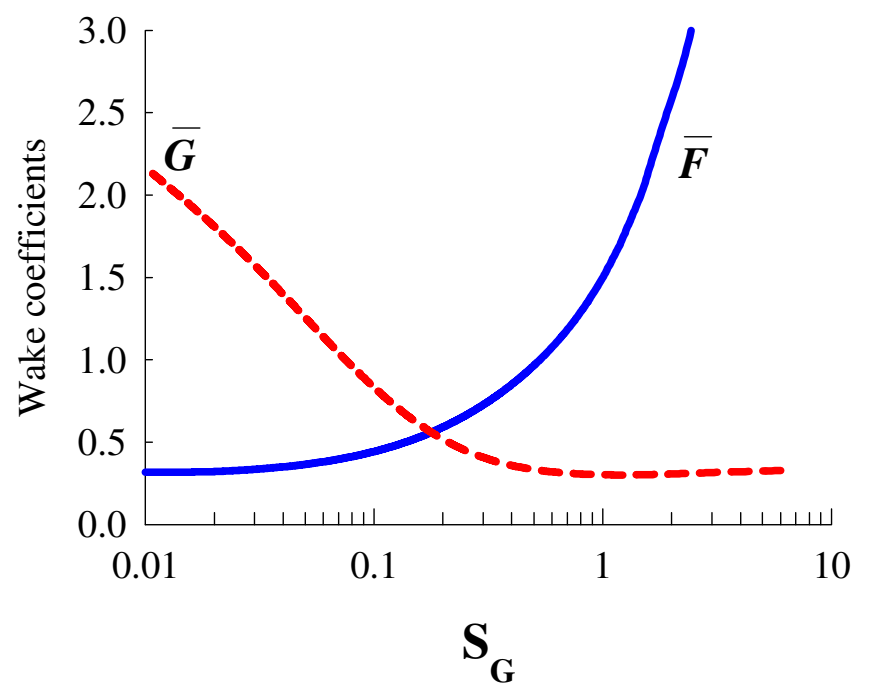

Figure 2 Variation of empirical wake coefficients with mass-damping parameter $S_{G}$ 


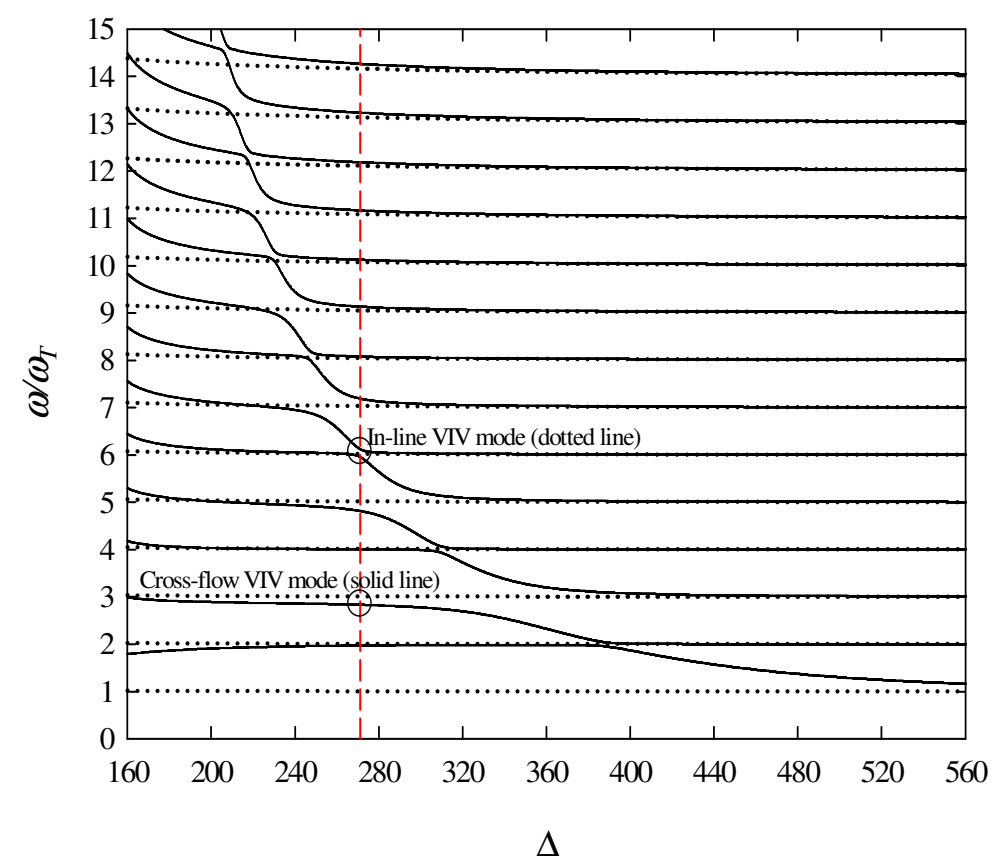

Figure 3 Variation of riser natural frequencies with tensioned-beam parameter $\Delta$ 

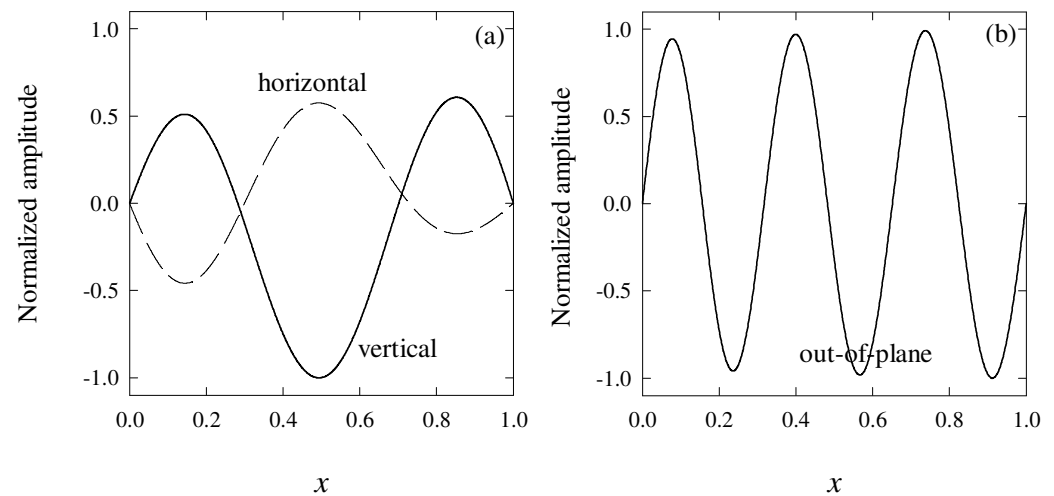

Figure 4 Considered (a) $2^{\text {nd }}$ in-plane and (b) $6^{\text {th }}$ out-of-plane modes for cross-flow and in-line VIV, respectively 

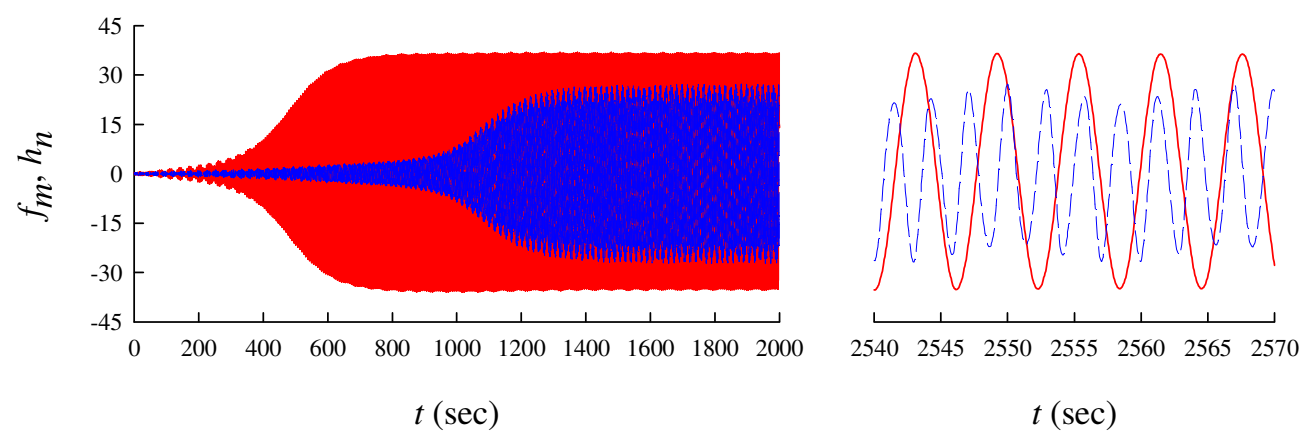

Figure 5 Time histories of riser displacement coordinates and associated with cross-flow $\left(f_{m}\right.$, red solid line) and in-line ( $h_{n}$, blue dotted line) VIV 


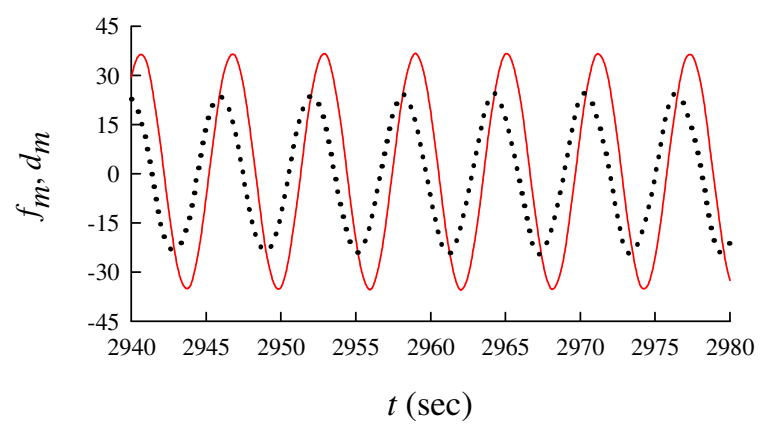

(a)
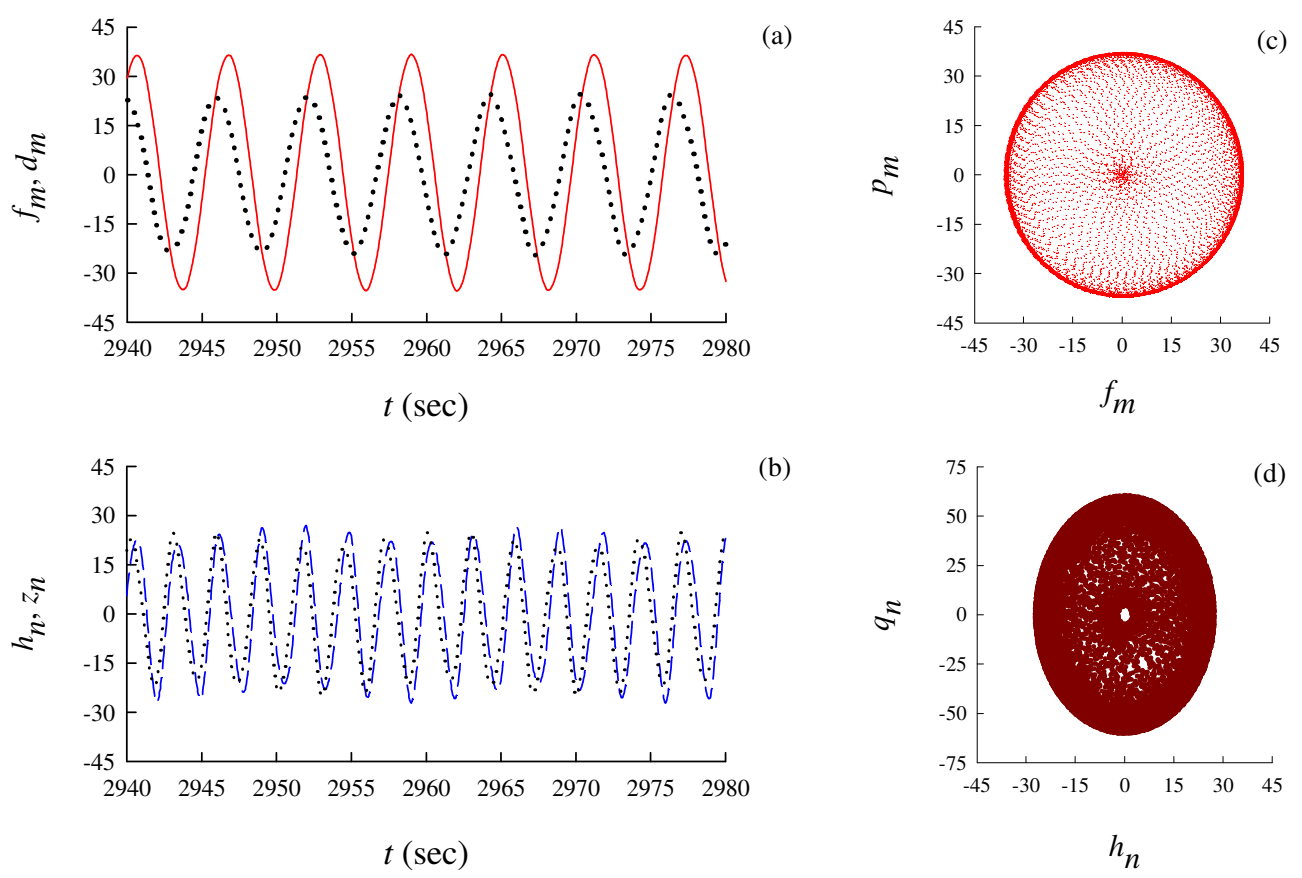

(b)

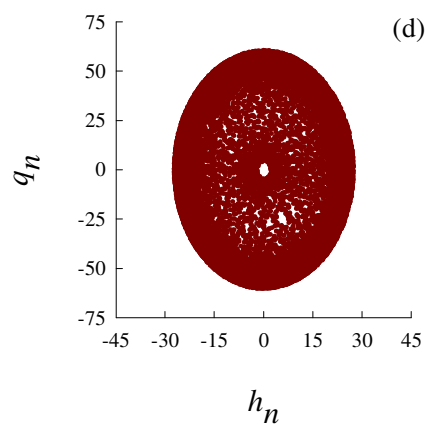

Figure 6 (a) Cross-flow VIV (solid line) and lift force (dotted line) responses, (b) In-line VIV (dashed line) and drag force (dotted line) responses, with corresponding phase portraits of riser motion $\left(f_{m}-p_{m}\right.$ and $\left.h_{n}-q_{n}\right)$ in (c) and (d) 
Click here to download Figure: Fig. 7.pdf

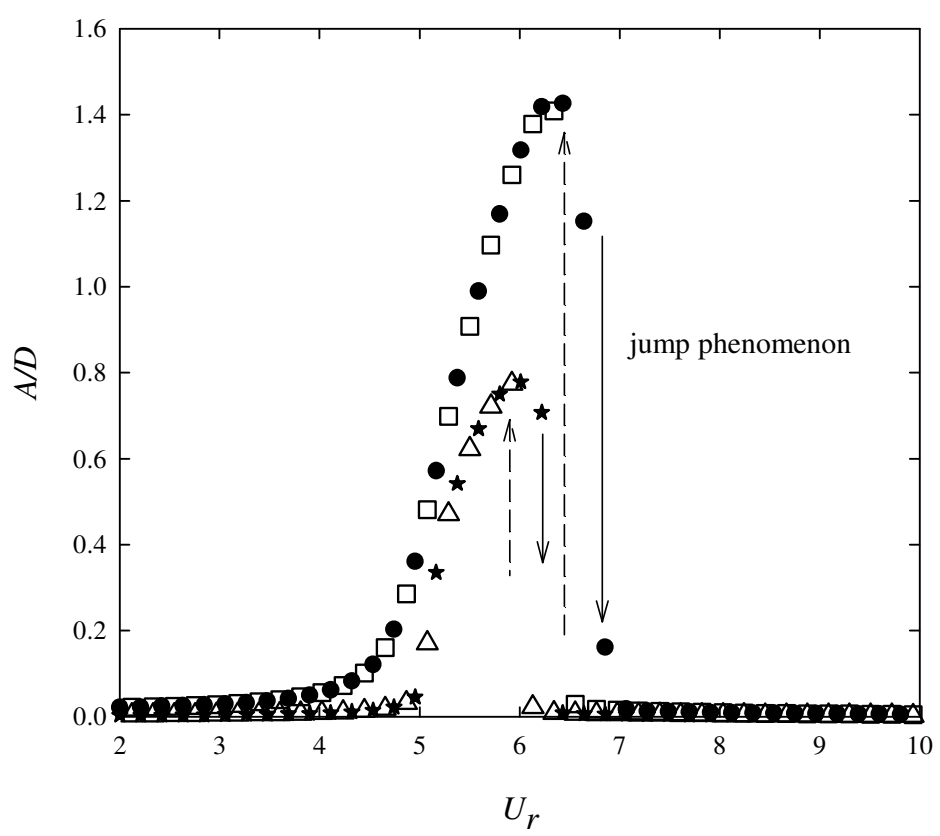

Figure 7 Maximum amplitude responses due to cross-flow (circles, squares) and in-line (stars, triangles) VIV of SCR, with increasing $V$ (circles, stars) or decreasing $V$ (squares, triangles) 

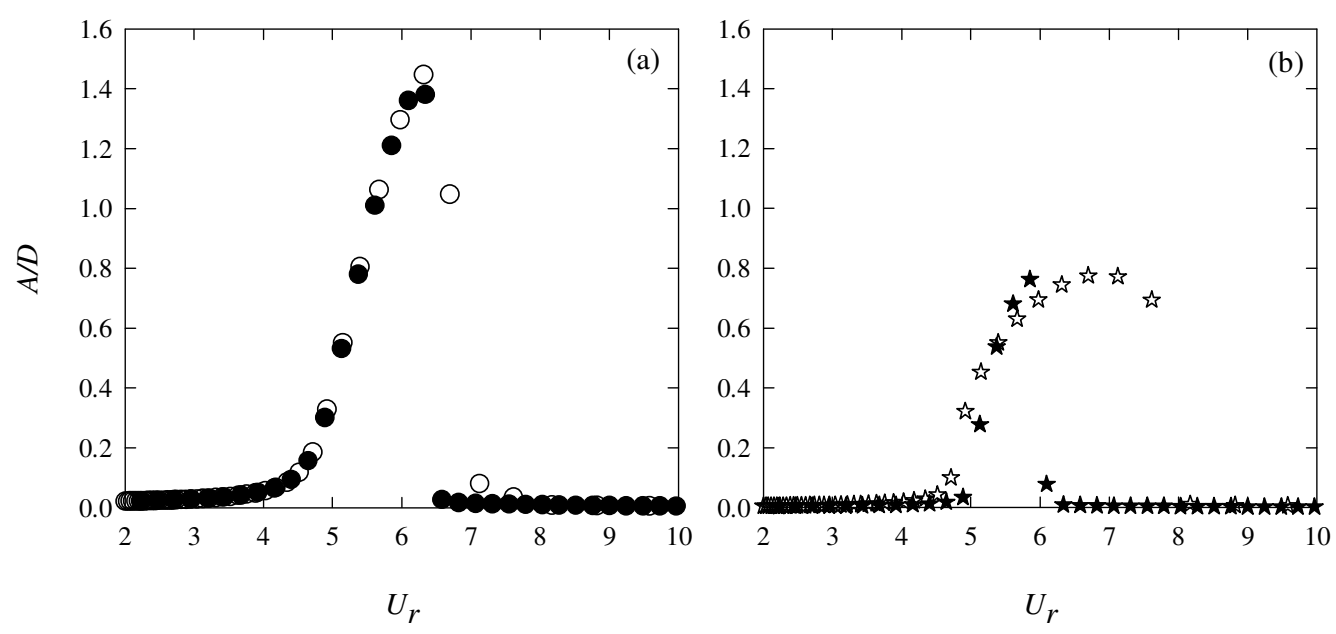

Figure 8 Maximum amplitude responses due to (a) cross-flow and (b) in-line VIV of SCR, with varying $\omega_{s}$ (filled symbols) or varying $\omega_{m}$ and $\omega_{n}$ (open symbols) 
Click here to download Figure: Fig. 9.pdf
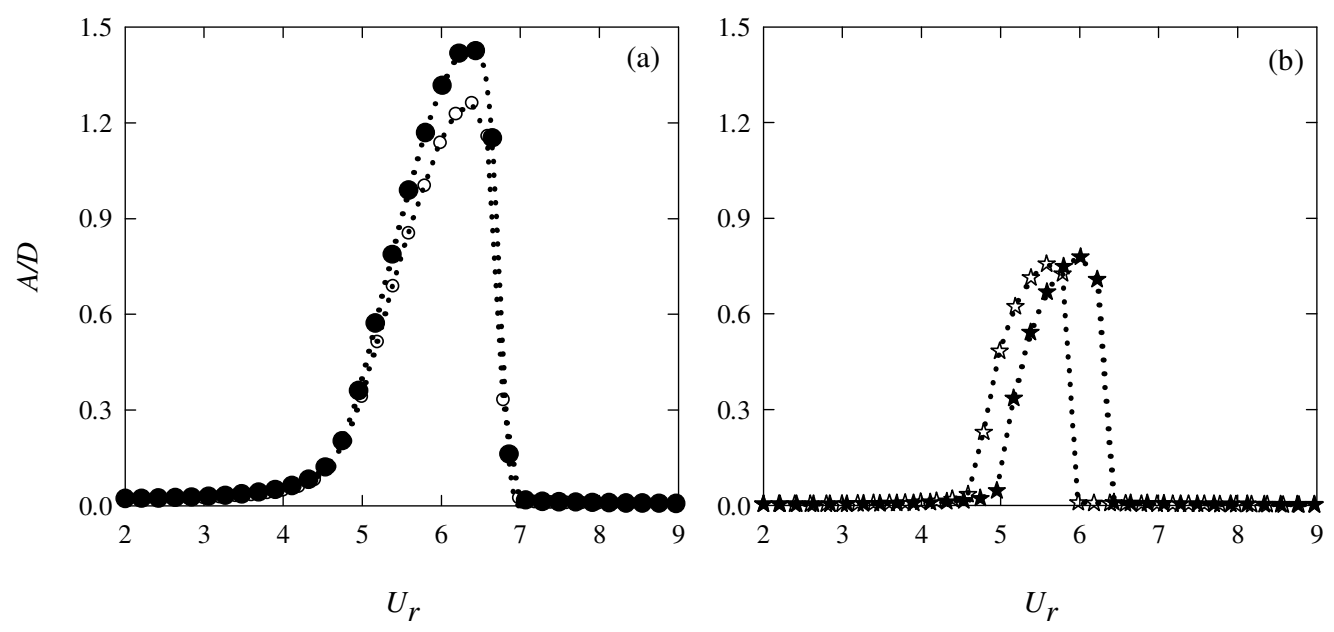

Figure 9 Maximum amplitude responses due to (a) cross-flow and (b) in-line VIV: SCR (filled symbols), TTR (open symbols) 
Fig.10

Click here to download Figure: Fig. 10.pdf
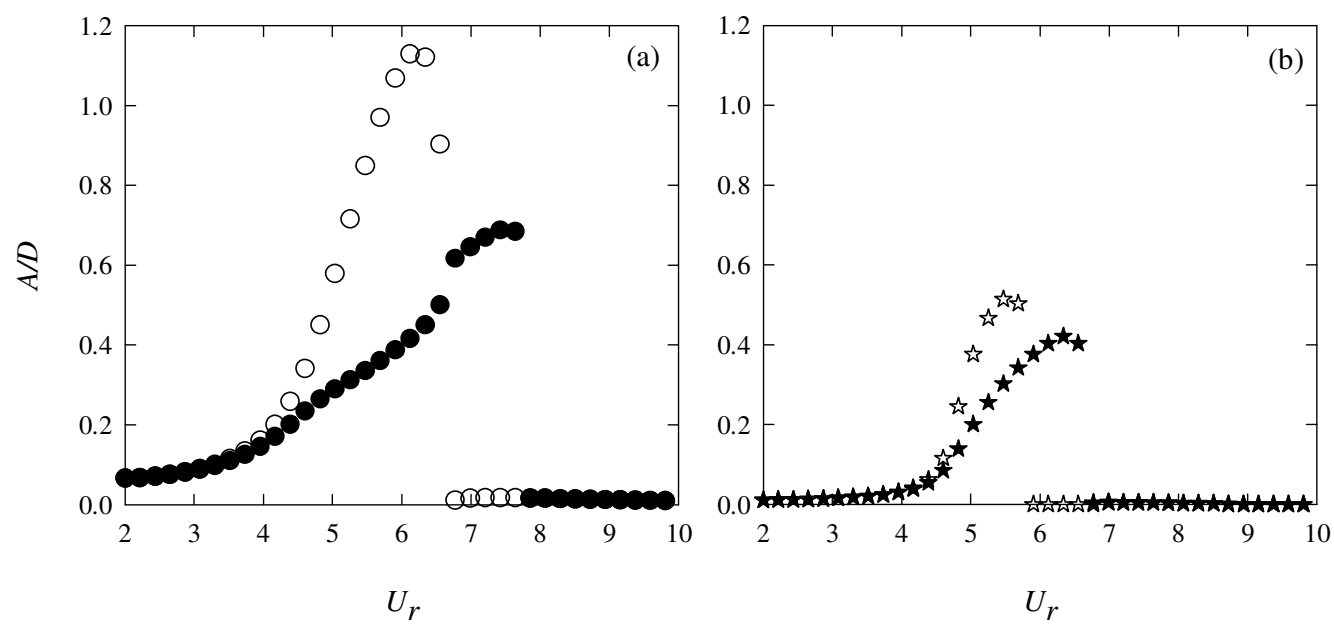

Figure 10 Maximum amplitude responses due to (a) cross-flow and (b) in-line VIV of SCR, with geometrically nonlinear (filled symbols) and linear (open symbols) modelling 
Fig.11

Click here to download Figure: Fig. 11.pdf

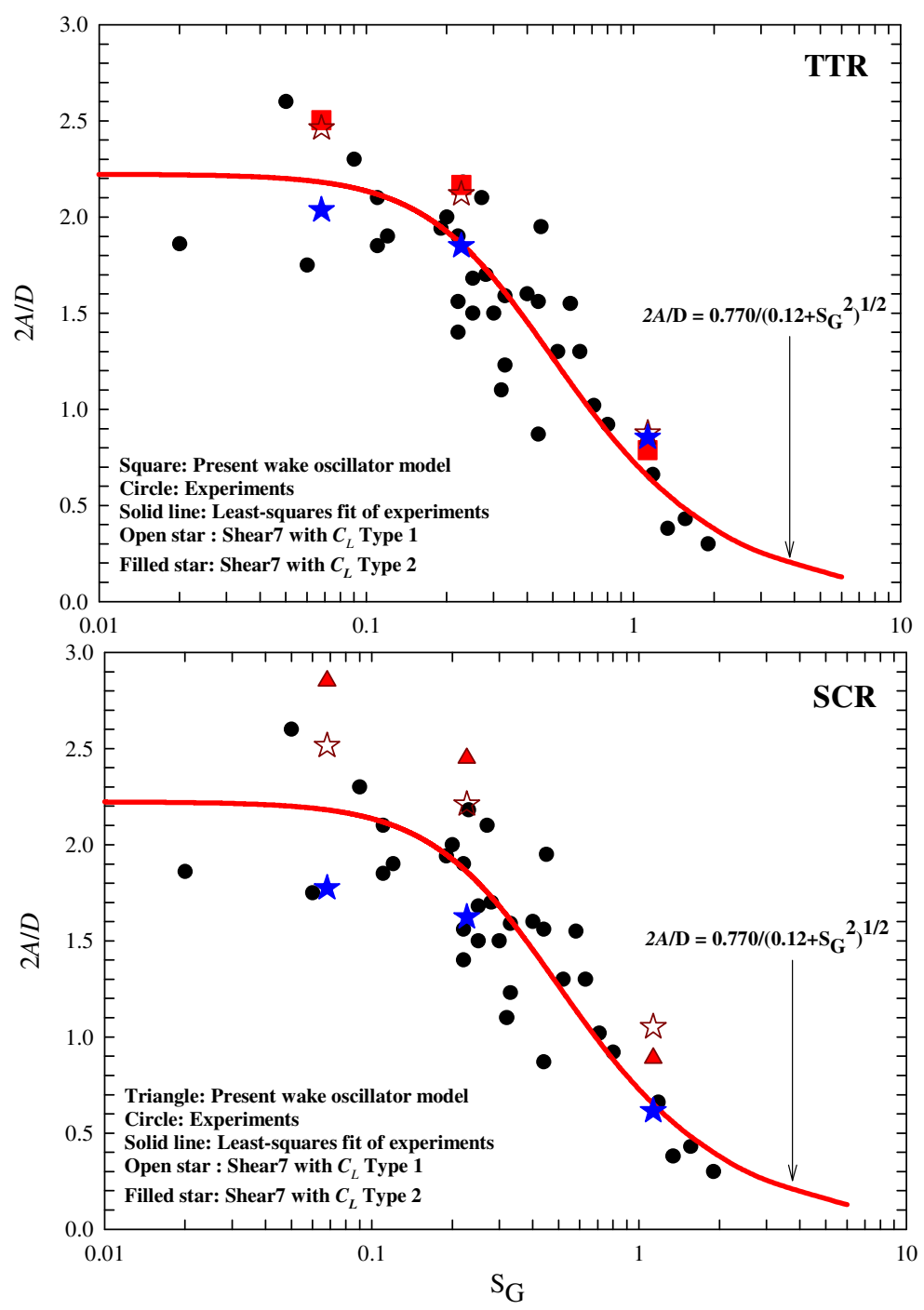

Figure 11 A comparison of maximum cross-flow VIV amplitudes during lock-in of TTR and SCR with some experimental data and Shear7 results 
Table 1 A comparison of maximum $A / D$ due to cross-flow and in-line VIV for SCR with $\Delta=272$ involving different excited modes

\begin{tabular}{|c|c|c|}
\hline Cross-flow $:$ In-line Modes & Cross-flow $A / D$ & In-line $A / D$ \\
\hline $1: 4$ & 1.142 & 0.775 \\
\hline $2: 6$ & 1.426 & 0.780 \\
\hline $3: 8$ & 1.326 & 0.759 \\
\hline $4: 10$ & 1.423 & 0.742 \\
\hline
\end{tabular}

Table 2 A comparison of maximum $A / D$ due to cross-flow and in-line VIV for SCRs having different tensioned-beam parameters $\Delta$ and sag-to-span ratios

\begin{tabular}{|c|c|c|c|}
\hline$\Delta$ & Sag/span & Cross-flow $A / D$ & In-line $A / D$ \\
\hline 200 & 0.162 & 1.389 & 0.787 \\
\hline 340 & 0.052 & 1.343 & 0.771 \\
\hline 520 & 0.022 & 1.277 & 0.751 \\
\hline
\end{tabular}

